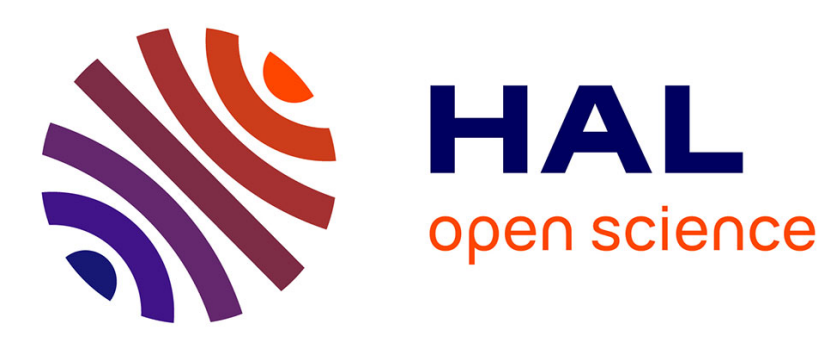

\title{
Probabilistic Schedulability Analysis for Fixed Priority Mixed Criticality Real-Time Systems
}

Yasmina Abdeddaïm, Maxim Dorin

\section{To cite this version:}

Yasmina Abdeddaïm, Maxim Dorin. Probabilistic Schedulability Analysis for Fixed Priority Mixed Criticality Real-Time Systems . Design, Automation and Test in Europe - DATE 2017, Mar 2017, Lausanne, Switzerland. hal-01583159

\section{HAL Id: hal-01583159 \\ https://hal.science/hal-01583159}

Submitted on 6 Sep 2017

HAL is a multi-disciplinary open access archive for the deposit and dissemination of scientific research documents, whether they are published or not. The documents may come from teaching and research institutions in France or abroad, or from public or private research centers.
L'archive ouverte pluridisciplinaire HAL, est destinée au dépôt et à la diffusion de documents scientifiques de niveau recherche, publiés ou non, émanant des établissements d'enseignement et de recherche français ou étrangers, des laboratoires publics ou privés. 


\section{Probabilistic Schedulability Analysis for Fixed Priority Mixed Criticality Real-Time Systems}

\author{
Yasmina Abdeddaïm \\ Université Paris-Est, LIGM, ESIEE Paris, France
}

\author{
Dorin Maxim \\ University of Lorraine, LORIA/INRIA, France
}

\begin{abstract}
In this paper we present a probabilistic response time analysis for mixed criticality real-time systems running on a single processor according to a fixed priority pre-emptive scheduling policy. The analysis extends the existing state of the art probabilistic analysis to the case of mixed criticalities, taking into account both the level of assurance at which each task needs to be certified, as well as the possible criticalities at which the system may execute. The proposed analysis is formally presented as well as explained with the aid of an illustrative example.
\end{abstract}

\section{INTRODUCTION}

In this paper we propose a probabilistic analysis for Mixed Criticality Real-Time Systems (MCRTS). A MCRTS incorporates several functionalities of different criticalities on the same architecture. The highest criticality functionalities are usually related to safety critical applications and need to fulfill strict certification requirements. It is important to note that lower critical functionalities are still relevant for the good functioning of the system [1] and this is a key observation for our approach.

We introduce a probabilistic mixed criticality model where every task is of a certain criticality and the worst-case execution time (WCET) of a task is a discrete random variable. The set of possible execution times of every task are grouped into sets of WCETs of different criticalities depending on their probability of occurrence. Larger execution time values are less likely to occur, but if they do occur they may be indicative of an erroneous event and so the criticality of the system changed to a higher level. When the citicality of the system is high, the guarantees required from lesser criticality tasks may be decreased so that higher criticality tasks may fulfill their requirements. The main difference between our work and the state of the art is the fact that in our work tasks are not evicted from the system, but instead the timing constraints imposed on them are gradually decreased (with the increase of the systems criticality) so that they still provide a minimal quality of service.

The first work dealing with real-time scheduling for mixed criticality systems is that of Vestal [2]. The model presented in [2] is based on the conjecture that the higher is the degree of criticality at which a task is designed, the more conservative is its worst-case execution time (WCET). This means that tasks have several WCET estimates, one per level of criticality of the system, and a mixed criticalty system is correct if every task respects its timing guarantees when all the WCET values that can impact on the schedulability of that task do not exceed their WCET estimates at the level of criticality of that task. Since then, many papers dealing with the mixed criticality scheduling problem have been published. For more details on the mixed criticality scheduling problem a complete review can be found in [3]. Further-on we present related work from the Probabilistic Analysis domain and work that is at the intersection of mixed criticality and probabilistic analysis.

Probabilistic timing analysis models task parameters as random variables (e.g. the task's WCET, Minimum Inter-arrival Time (MIT)). For WCET analysis multiple paths have been explored, starting in 1995 when [4] introduced an analysis for tasks that have periodic releases but variable execution requirements. The algorithm called Probabilistic Time Demand Analysis (PTDA) is based on a bound of the processor demand of higher priority tasks and hence it is highly pessimistic. The next step towards an exact probabilistic analysis was made by [5] with the introduction of the Stochastic Time Demand Analysis (STDA) for tasks that have probabilistic execution times, computing a lower bound on the probability that jobs of each task will meet their respective deadlines. Later on, [6] refined STDA into an exact analysis for real-time systems that have random execution times, represented as general random variables. In this work we extend the probabilistic response time analysis of [6] to the case of MCRTS.

Existing probabilistic analyses for real-time systems are not well suited for the analyses of MCRTSs, as they do not take into account the possible criticalities of the system and the fact that permitted failure proabilities might change when the criticality of the system changes.

A first step towards a probabilistic analysis for MCRTSs is [7], where the authors consider a dual criticality model consisting of implicit-deadline sporadic independent tasks where a probability that no job exceeds its low criticality WCET estimate is assigned to every high criticality task. In this model a system is probabilistically schedulable in the strong sense if the probability of missing a deadline (for any task) does not exceed a given threshold, while it is probabilistically schedulable in the weak sense if the set of high criticality tasks does not exceed their respective deadlines. A schdeulability analysis of an EDF-based (earliest deadline first) algorithm is proposed. Using this analysis a system that is deemed unschedulable using classical mixed criticality analysis can be deemed schedulable in a probabilistic sense. In [8] the authors introduce the notion of probabilistic C-Space and give some intuitions concerning the way it can be used for the 
probabilistic sensitivity analysis of a mixed criticality system.

Our approach has some similarities with the analysis of [7] in the fact that it extends the probabilistic analysis to the case of mixed criticalities, however the analysis we propose is applicable for fixed priority pre-emptive systems (rather then EDF) and for systems with more than two criticality levels. Another way in which our contribution differs from that of [7] is that in our model jobs are not dropped to increase the schedulability of other tasks, but all tasks need to be verified to be within certain thresholds of failure probabilities depending on their criticalities and the criticality of the system.

Organization of the paper: We continue the paper with the description of the system model in Section II and the problem description in Section III. Then in Section IV we present the main contribution of our work, which is a theoretical response time analysis for mixed criticality real-time systems. To show how the analysis can be applied on a task-set we present an ilustrative example in Section V. Finally we conclude the paper in Section VI.

\section{Model And TERMinology}

We model the mixed criticality real-time system to be analysed as a set of tasks $\Gamma=\left\{\tau_{1}, \ldots, \tau_{n}\right\}$ sorted in an increasing order of priority, from the lowest priority $\left(\tau_{1}\right)$ to the highest priority $\left(\tau_{n}\right)$, scheduled according to a pre-emptive fixed priority policy on a single processor.

In adition to the set of tasks the system is also characterized by a set of criticalities $\Omega=\left\{L_{1}, \ldots, L_{m}\right\}$. We consider $\Omega$ to be sorted from lowest criticality $L_{1}$ to highest criticality $L_{m}$. To every criticality level a maximum-probability of failure threshold is attributed by a deciding entity (e.g. system designer, certification authority). We denote this threshold by $p^{L_{i}}$ for criticality $L_{i}$. Intuitively a higher criticality implies a more stringent probability threshold.

Each task $\tau_{i}$ is characterized by a tuple $\tau_{i}=\left(\chi_{i}, \mathcal{C}_{i}, T_{i}, D_{i}\right)$ such that $\chi_{i} \in \Omega$ represents its criticality, $\mathcal{C}_{i}{ }^{1}$ represents its probabilistic worst-case execution time (pWCET), $T_{i}$ represents its minimum inter arrival time and $D_{i}$ represents its relative deadline. The criticality of each task is defined by the system designer at design-time.

The pWCET $\mathcal{C}_{i}$ of a task $\tau_{i}$ is given as a discrete random variable with a sample space $S_{\mathcal{C}_{i}}$ and a probability mass function (pmf) $p_{\mathcal{C}_{i}}$ where $p_{\mathcal{C}_{i}}(c)$ is equal to $P\left\{\mathcal{C}_{i}=c\right\}$, the probability that $\mathcal{C}_{i}$ is equal to $c$. The probability mass function of $\mathcal{C}_{i}$ is represented as:

$$
p_{\mathcal{C}_{i}}=\left(\begin{array}{lll}
\mathcal{C}_{i, 1} & \ldots & \mathcal{C}_{i,\left|S_{\mathcal{C}_{i}}\right|} \\
P\left\{\mathcal{C}_{i}=C_{i, 1}\right\} & \ldots P\left\{\mathcal{C}_{i}=C_{i,\left|S_{\mathcal{C}_{i}}\right|}\right\}
\end{array}\right)
$$

We consider pWCET distributions to be known and their calculation is beyond the scope of this paper. The interested reader may refer to [9] and [10] for further reading on this topic.

Two random variables $\mathcal{X}$ and $\mathcal{Y}$ are (probabilistically) independent if they describe two events such that the outcome

\footnotetext{
${ }^{1}$ Throughout the paper we use calligraphic typeface to denote random variables
}

of one event does not have any impact on the outcome of the other. As stated in [9], since we consider probabilistic worst-case values (for WCETs), the random variables are (probabilistically) independent.

Let $c d f(c)=P\left\{\mathcal{C}_{i} \leq c\right\}$ be the cumulative distribution function of the pWCET random variable. A WCET outcome $C_{i, k}$ of the $p W C E T \mathcal{C}_{i}$ is of criticality $L_{j}$ if and only if the probability that the pWCET exceeds $C_{i, k}\left(P\left\{\mathcal{C}_{i}>C_{i, k}\right\}=\right.$ $1-c d f\left(C_{i, k}\right)$ ) does not exceed the probability failure threshold $p^{L_{j}}$. The subset of WCETs of criticality $L_{j}$ of task $\tau_{i}$ is denoted by $S_{\mathcal{C}_{i}}^{L_{j}}$ and is given by the following Equations:

$$
\begin{gathered}
S_{\mathcal{C}_{i}}^{L_{j}}=\left\{C_{i, k} \in S_{\mathcal{C}_{i}} / p^{L_{j+1}}<1-c d f\left(C_{i, k}\right) \leq p^{L_{j}}\right\} \\
\text { if } \quad 1 \leq j<m \text { and } \\
\quad S_{\mathcal{C}_{i}}^{L_{m}}=\left\{C_{i, k} \in S_{\mathcal{C}_{i}} / 1-c d f\left(C_{i, k}\right) \leq p^{L_{m}}\right\} .
\end{gathered}
$$

Intuitively, Equations 2 and 3 describe the fact that the pWCET distribution of a task can be split into $m$ pieces (i.e. partial distributions), one piece for each criticality level. One or several such partial distributions may be void. The boundary of each piece is given by the minimal and maximal WCET values that the task can exhibit in a certain criticality level of the system, i.e. larger values would push the system into a higher criticality mode.

The representative WCET of a task $\tau_{i}$ in criticality level $L_{j}$, noted $C_{i}\left(L_{j}\right)$, is the largest WCET outcome of criticality less than or equal to $L_{j}$. The representative WCET $C_{i}\left(L_{j}\right)$ is equal to zero if all the WCET outcomes of task $\tau_{i}$ are of higher criticality than $L_{j}$.

We define the criticality mode of the system ${ }^{2}$ as the smallest criticality such that no task $\tau_{i}$ executes for more than its representative WCET estimate at this level of criticality.

We note by:

$$
p_{\mathcal{C}_{i}}^{\leq L_{h}}=\left(\begin{array}{lll}
\mathcal{C}_{i, 1} & \ldots & \mathcal{C}_{i}\left(L_{h}\right) \\
p_{\mathcal{C}_{i}}\left(\mathcal{C}_{i, 1}\right) & \ldots & p_{\mathcal{C}_{i}}\left(\mathcal{C}_{i}\left(L_{h}\right)\right)
\end{array}\right)
$$

to be the partial mass function of $p_{\mathcal{C}_{i}}$ restricted to WCET outcomes of criticality less than or equal to the criticality $L_{h}$. If the set of WCET oucomes of critiality less than or equal to $L_{h}$ is empty, then $p_{\mathcal{C}_{i}}^{\leq L_{h}}(r)=0, \forall r \in \mathbb{N}$.

Each task $\tau_{i}$ generates an infinite number of jobs noted $\tau_{i, k}$. All jobs are assumed to be independent of other jobs of the same task and those of other tasks. The execution time of a job $\tau_{i, k}$ is denoted by $c_{i, k}$. We use $h p(i)$ to be the indexes of tasks of higher or equal priority than $\tau_{i}$.

The response time analysis for probabilistic real-time systems makes use of the convolution operator, which is a way of summing up two independent random variables and it is formally defined as follows:

Definition 1. The probability mass function $p_{\mathcal{Z}}$ of the sum $\mathcal{Z}$ of two (probabilistically) independent random variables $\mathcal{X}$ and $\mathcal{Y}$ is the convolution $p_{\mathcal{X}} \otimes p_{\mathcal{Y}}$ where $P\{\mathcal{Z}=z\}=$ $\sum_{k=-\infty}^{k=+\infty} P\{\mathcal{X}=k\} P\{\mathcal{Y}=z-k\}$.

\footnotetext{
${ }^{2}$ In the rest of the paper, criticality level denoted by $L \in\left\{L_{1}, \ldots, L_{m}\right\}$ is used for tasks' criticality, and criticality mode is used for system criticality
} 
A probabilistic analysis for (non-mixed criticality) real-time tasks where the WCET is a discrete random variable has been proposed in [6]. In this analysis, the worst-case response time probability mass function $p_{\mathcal{R}_{i}}$ of task $\tau_{i}$ is computed using the following equation (for more details please refer to [6]):

$$
p_{\mathcal{R}_{i}}=\mathcal{B}_{i} \otimes p_{\mathcal{C}_{i}} \otimes \mathcal{I}_{i}
$$

where $\mathcal{B}_{i}=\left(\bigotimes_{j \in h p(i)} p_{\mathcal{C}_{j}}\right)$ is the accumulated execution time requirements of all higher priority tasks that are instantiated at the same time as the task under analysis and $\mathcal{I}_{i}$ is the iterative convolution of jobs that may preempt the task under analysis. Note that Equation 5 is computed as if jobs would still continue to execute past their deadlines since this is an upper-bound over the case when jobs are stopped at their deadline. The exact analysis for the case when jobs are dropped at deadline is still an open problem, but the no-drop analysis is a tight over-approximation for the drop analysis and its tightness is related to the probabilities of missing deadlines as this is the difference between the two analyses.

\section{PROBLEM DESCRIPTION}

In this paper we are interested in the pre-emptive fixed priority scheduling problem of probabilistic mixed criticality real-time systems running on a single processor. Under this mixed criticality probabilistic model introduced in Section II a schedule is considered feasible if and only if: when the criticality mode of the system is not larger than the criticality of a task, each task respects the probability of failure threshold specified for its criticality level. The idea is that all the tasks are certified at their own criticality level, but the lowest criticality tasks should not disturb the highest criticality ones when the criticality mode of the system increases. That is, when the criticality of the system increases, lower criticality tasks are no longer constrained to respect a stringent probability failure threshold, but instead the threshold is replaced with a more relaxed one so to ensure minimal quality of service while not hindering the timely execution of higher criticality tasks.

We consider that a task exhibits a failure if one of its jobs misses it deadline, consequently, the probability failure of a task is equal to its probability of deadline miss. In this work we consider that: (1) if a job exceeds the maximum WCET estimate corresponding to its criticality level it is not stopped and (2) a job is evacuated from the system if and only if it misses its deadline. The problem addressed in this paper is the computation of tasks' deadline miss probabilities (DMP) in order to verify that failure thresholds are respected and the system can be declared schedulable. To this extend we propose an analysis to compute response time distributions for tasks. Out of these response time distributions we can then extract deadline miss probabilities.

\section{PMC SCHEDUlabILITy ANALYSIS}

\section{A. Response Time Analysis}

In this section we introduce our probabilistic analysis for MCRTS, which we will denote by PMC (Probabilistic Mixed Criticality) in the rest of the paper.
The Probabilistic Mixed Criticality (PMC) response time analysis computes the partial probability mass functions $p_{\mathcal{R}_{i}}^{L_{h}}$ of the worst-case response time of task $\tau_{i}$ when the criticality mode of the system is $L_{h}$.

Given $p_{\mathcal{R}_{i}}$, the probability mass function of $\mathcal{R}_{i}$, one can decompose the worst-case response time probability mass function into partial functions as follows,

$$
p_{\mathcal{R}_{i}}=\left(\begin{array}{c|l|l}
p_{\mathcal{R}_{i}}^{L_{1}} & \ldots & p_{\mathcal{R}_{i}}^{L_{m}}
\end{array}\right)
$$

where

$$
p_{\mathcal{R}_{i}}^{L_{h}}=\left(\begin{array}{ccc} 
& r & \\
& p_{\mathcal{R}_{i}}^{L_{h}}(r) & \ldots
\end{array}\right)
$$

is a partial function of $p_{\mathcal{R}_{i}}$ with $p_{\mathcal{R}_{i}}^{L_{h}}(r)$ is the probability that the worst-case response time of $\tau_{i}$ is equal to $r$ when,

1) none of the jobs that are active in the interval $[0, r[$ executes more than $C_{i}\left(L_{h}\right)$, i.e. their respective representative WCET in the criticality mode $L_{h}$ and,

2) if $h>1$, there exists at least an active job in the interval $\left[0, r\left[\right.\right.$ that executes more than $C_{i}\left(L_{h-1}\right)$, i.e. its representative WCET at the criticality mode $L_{h-1}$.

More formally,

$$
\begin{aligned}
p_{\mathcal{R}_{i}}^{L_{h}}(r)= & P\left\{\mathcal { R } _ { i } = r \text { and } \forall \tau _ { j , k } \in \left[0, r\left[, c_{j, k} \leq C_{j}\left(L_{h}\right)\right.\right.\right. \\
& \text { and if } h>1, \exists \tau_{j, k} \in\left[0, r\left[\text { s.t. } c_{j, k}>C_{j}\left(L_{h-1}\right)\right\} .\right. \\
= & P\left\{\mathcal{R}_{i}=r \text { and } L=L_{h}\right\} \\
& \text { with } L \text { the criticality mode of the system }
\end{aligned}
$$

Note that,

$$
\sum_{L_{h} \in L} \sum_{r \in S_{\mathcal{R}_{i}}^{L_{h}}} p_{\mathcal{R}_{i}}^{L_{h}}(r)=1
$$

where $S_{\mathcal{R}_{i}}^{L_{h}}$ is the subset of outcomes of $\mathcal{R}_{i}$ in the case where the criticality mode of the system is $L_{h}$.

We emphasize that this partitioning of the response time distribution of task $\tau_{i}$ according to the different criticality modes is not a simple slicing of the response time distribution into $m$ parts, but there can exist overlapping among these parts and each part is computed by the analysis. The coalescing of all the parts forms the complete response time distribution of the task regardless of the criticality mode of the system. The coalesced distribution is also the result returned by the analysis of [6] which does not take into account the different criticalities of tasks nor of the system. The computation of the partial response-times probability mass functions $p_{\mathcal{R}_{i}}^{L_{h}}$ of a task $\tau_{i}$ is formalised in Algorithm 1. In step 1 the algorithm computes for every criticality $L_{h}$ the partial response time probability mass function when the criticaliy mode is less than or equal to $L_{h}$, for each $L_{h}$. Then in step 2 the partial response time probability mass functions for each criticality mode are computed. Steps 1 and 2 are detailed below.

Step 1: Compute the partial probability mass function $p_{\mathcal{R}_{i}}^{\leq L_{h}}$ using Equation 6 below:

$$
p_{\mathcal{\mathcal { R }}_{i}}^{\leq L_{h}}=\bigotimes_{j \in h p(i)} p_{\overline{\mathcal{C}}_{j}}^{\leq L_{h}} \otimes p_{\mathcal{\mathcal { C }}_{i}}^{\leq L_{h}} \otimes \mathcal{I}_{i}^{\leq L_{h}}
$$




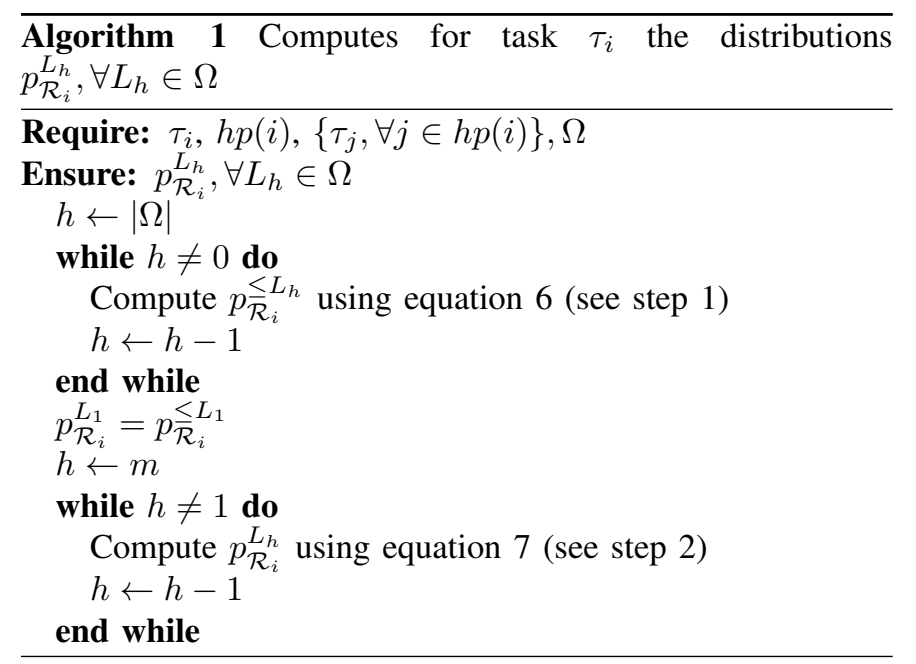

Equation 6 is similar to Equation 5 where partial probability mass functions $p_{\mathcal{C}_{i}}^{\leq L_{h}}$ are used rather than the complete probability mass functions.

Step 2: Compute the partial probability mass function $p_{\mathcal{R}_{i}}^{L_{h}}$.

In this step we compute $p_{\mathcal{R}_{i}}^{L_{h}}$ the partial probability mass function of $p_{\mathcal{R}_{i}}$ when the criticality mode is equal to $L_{h}$ using equation 7 :

$$
p_{\mathcal{R}_{i}}^{L_{h}}=\left\{\begin{array}{l}
p_{\mathcal{R}_{i}}^{\leq L_{1}} \text { if } h=1 \\
p_{\mathcal{R}_{i}}^{\leq L_{h}} \ominus p_{\mathcal{R}_{i}}^{\leq L_{h-1}} \text { if } h>1
\end{array}\right.
$$

where $f \ominus g(x)=f(x)-g(x) \forall x \in \mathbb{N}$.

Note that the $\ominus$ operator is well defined in this case, as the (partial)distribution $p_{\mathcal{R}_{i}}^{\leq L_{h-1}}$ to be subtracted is a subset of the (partial)distribution $p_{\mathcal{\mathcal { R }}_{i}}^{\leq L_{h}}$, i.e. the sample space of $p_{\mathcal{R}_{i}}^{\leq L_{h-1}}$ is included in the sample space of $p_{\mathcal{R}_{i}} L_{h}$ as a result of the partial distributions used in Step 1. Below is an example of how this operators works:

$\left(\begin{array}{cccc}1 & 2 & 3 & 4 \\ 0.4 & 0.2 & 0.1 & 0.1\end{array}\right) \ominus\left(\begin{array}{ccc}1 & 2 & 3 \\ 0.4 & 0.1 & 0.1\end{array}\right)=\left(\begin{array}{cc}2 & 4 \\ 0.1 & 0.1\end{array}\right)$

Proposition 1. The probability that "the worst-case response time of task $\tau_{i}$ is equal to $r$ and the criticality mode of the system is equal to $L_{h}$ " is equal to $p$ if and only if $p_{\mathcal{R}_{i}}^{L_{h}}(r)=p$ where $p_{\mathcal{R}_{i}}^{L_{h}}(r)$ is computed using Equation 7.

Proof. By definition, $p_{\mathcal{R}_{i}}^{\leq L_{h}}(r)$ is the probability that the response time of task $\tau_{i}$ is equal to $r$ and the criticality of the system is less than or equal to $L_{h}$. This probability is equal to the sum of the probabilities that the response time is equal to $r$ computed in every criticality mode of the system less than or equal to $L_{h}$, thus

$$
p_{\mathcal{R}_{i}}^{\leq L_{h}}(r)=\sum_{j=1 \ldots h} P\left\{\mathcal{R}_{i}=r \text { and } L=L_{j}\right\}
$$

and similarly,

$$
p_{\mathcal{R}_{i}}^{\leq L_{h-1}}(r)=\sum_{j=1 \ldots h-1} P\left\{\mathcal{R}_{i}=r \text { and } L=L_{j}\right\}
$$

The partial distribution $p_{\mathcal{R}_{i}}^{\leq L_{h-1}}(r)$ is a subset of $p_{\mathcal{R}_{i}}^{\leq L_{h}}(r)$.
Using Equation 7, we have $p_{\mathcal{R}_{i}}^{L_{h}}(r)=p_{\mathcal{\mathcal { R }}_{i}}^{L_{h}}(r)-$ $p_{\mathcal{R}_{i}}^{\leq L_{h-1}}(r)=\sum_{j=1 \ldots h} P\left\{\mathcal{R}_{i}=r\right.$ and $\left.L=L_{j}\right\}-\sum_{j=1 \ldots h-1} P\left\{\mathcal{R}_{i}=\right.$ $r$ and $\left.L=L_{j}\right\}$ and thus,

$$
p_{\mathcal{R}_{i}}^{L_{h}}(r)=P\left\{\mathcal{R}_{i}=r \text { and } L=L_{h}\right\}
$$

that is equal to the probability that the worst-case response time of task $\tau_{i}$ is equal to $r$ and the criticality mode of the system is equal to $L_{h}$.

\section{B. PMC Sufficient Schedulability Test}

Along with the PMC response-time analysis presented in the previous section we also propose a sufficient schedulability test which we present in this section. In the PMC feasibility test, for each criticality $L_{h}$, the deadline miss probability of a task $\tau_{i}$ needs to be less than or equal to the tasks' allowed failure probability threshold in criticality mode $L_{h}$ of the system.

Definition 2. (Task Deadline Miss Probability Per Criticality Mode). The deadline miss probability of a task $\tau_{i}$ in mode $L$, noted $D M P_{i}^{L}$, is the probability that task $\tau_{i}$ misses its deadline when the system is in criticality mode L. This probability is equal to:

$$
D M P_{i}^{L}=P\left\{\mathcal{R}_{i}^{L}>D_{i}\right\}
$$

where $\mathcal{R}_{i}^{L}$, as computed using Equation 7, is the random variable $\mathcal{R}_{i}$ restricted to outcomes in the case of a criticality mode of the system equal to $L$.

The tasks deadline miss probability constraint to a certain criticlity mode $L_{h}$ can be extracted from the partial response time mass functions of the task in the respective mode, using Equation 9 below.

$$
D M P_{i}^{L_{h}}=\sum_{r>D_{i}} p_{\mathcal{R}_{i}}^{L_{h}}(r)
$$

A sufficient feasibility test for PMC can be derived using Equation 9. If in each criticality mode $L$ the deadline miss probability of a task $\tau_{i}$ is less than or equal to the probability threshold imposed to the task in mode $L$, then the task is considered schedulable.

When the criticality mode of the system is greater than the criticality level of the task, the deadline miss probability threshold imposed on the task can be increased to 1 and in this case the task is allowed to miss all of its deadlines. Alternatively, the threshold can be increased to an intermediate value smaller than 1 , signifying that even though the task is less critical than the current mode of the system, it should still provide a certain quality of service, i.e. the probability of missing deadlines may be larger, but the task is not completely evicted from the system. The thresholds can be given by a standard or by a certification authority, or, alternatively, they can be decided upon by the system designer. An example of such schedulability thresholds is presented in Table III which also presents the degradation of tasks' failure thresholds as the criticality of the system increases. For example, a task of criticality 1 is allowed to have a DMP of 0.1 in system 


\begin{tabular}{|c|c|c|c|}
\hline & \multicolumn{3}{|c|}{ Criticality of the task } \\
\hline System's Criticality & $L_{1}$ & $L_{2}$ & $L_{3}$ \\
\hline \hline$L_{1}$ & 0.1 & 0.01 & 0.001 \\
$L_{2}$ & 0.5 & 0.01 & 0.001 \\
$L_{3}$ & 1 & 0.1 & 0.001 \\
\hline
\end{tabular}

Table III

Permitted Deadline Miss Probability Thresholds For the TASK-SET IN TABLE I

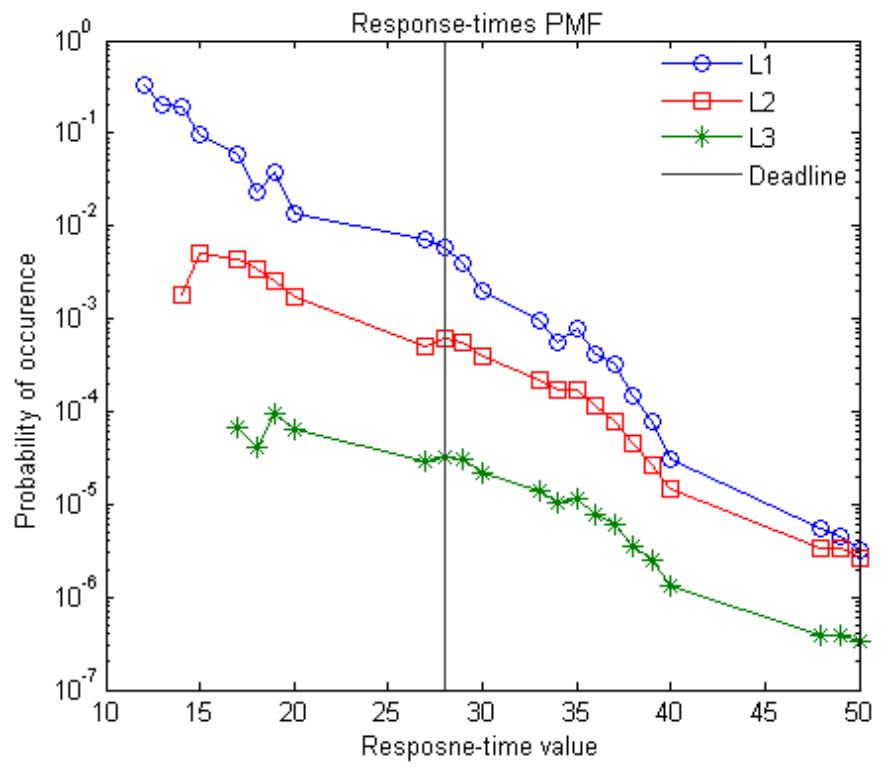

Figure 1. Response time partial distributions of task $\tau_{5}$ in different criticality modes of the system.

criticality mode $L_{1}$, a DMP of 0.5 in mode $L_{2}$ and a DMP of 1 in mode $L_{3}$, i.e. in $L_{3}$ a task of criticality $L_{1}$ is not required to provide any service.

A note on complexity: it is well known that probabilistic analyses are computationally intensive [11] and the analysis we propose in this paper makes no exception. Nevertheless there are efficient solutions in the literature to go around this problem, such as re-sampling [11]. We do not go into details about complexity and ways of reducing it, as we rather use simple task-sets to exemplify our technique and provide a proof of concept.

\section{ILlustrative EXAMPLE}

In order to provide an intuition of how the proposed analysis works we apply it on a simple tasks-set and show the results obtained. The task-set presented in Table I is composed of five tasks grouped in three levels of criticality $L_{1}, L_{2}$ and $L_{3}$. The pWCET distribution of each task has 6 values. The probability thresholds for each criticality level are $p^{L_{1}}=0.1, p^{L_{2}}=0.01$ and $p^{L_{3}}=0.001$ and they further degrade as shown in Table III. These threshold together with Equation 2 and Equation 3 are used to split the pWCET distribution of each task into the (various sized) partial distributions that are depicted in Table II.

For this example we will be analyzing only task $\tau_{5}$ as the analysis procedure is the same for all the other tasks. The system is scheduled according to deadline monotonic fixed priority preemptive scheduling policy, hence task $\tau_{5}$ is the lowest priority in the set. Also, task $\tau_{5}$ is considered to be of criticality $L_{2}$, an intermediate criticality in the system. From Table III we see that the schedulability constraints imposed to a task of criticality $L_{2}$ are as follows: if the system is in mode $L_{1}$ or $L_{2}$, which are normal modes from the point of view of a task of criticality $L_{2}$, i.e. neither it, nor any other task exceeded their largest WCET estimated for criticality $L_{2}$, then the task needs to function within a maximum allowed deadline miss probability of 0.01 . On the other hand if the system switches to criticality mode $L_{3}$ - which is an error mode from the point of view of a task of criticality $L_{2}$ meaning that one of the tasks in the system exceeded their largest WCET estimated for mode $L_{2}$ - its threshold is modified to 0.1. Intuitively, a lower criticality task would have larger allowed DMPs (i.e. thresholds) making it possible for the task to be placed at a lower priority level, and, consequently, more critical tasks may have higher priorities.

The response time partial distributions of task $\tau_{5}$ computed using our analysis are presented in Figure 1. We note that all curves were truncated at the value 50 as otherwise the plot would be too large and difficult to read and we mention only that maximal response time values of the task, in any mode, are infinitely large. That is, according to any deterministic analysis, task $\tau_{5}$ would never finish its execution in the worst case, meaning that the system would be deemed unschedulable (even for the lowest criticality mode). It is easy to see why this is the case, as the (deterministic) utilisations of the system are too large for it to be schedulable. For example the maximal utilisation, computed using the largest values of each (complete) pWCET distribution, is equal to 2.19. Even the minimal utilisation of the system, computed using the smallest values of each pWCET distribution, is equal to 0.56 , close to the schedulability limit of a fixed priority preemptive system.

We emphasize that the curves in Figure 1 are probability mass functions (PMFs) and not exceedence curves (i.e. 1$\mathrm{CDFs}$ ), and they show the probability that a specific response time value (read on the $\mathrm{X}$-axis) is observed during the execution of task $\tau_{5}$. These curves are decreasing exponentially (note the logarithmic Y-axis) as a result of the fact that the pWCETs of the example task-set are decreasing and so the larger response time values have exceedingly smaller probabilities of appearing. The decreasing pWCET distributions are characteristic of tasks of real systems, which are presumed to follow Gumbel distribution [12].

The deadline miss probability of task $\tau_{5}$ in each criticality mode of the system are computed by adding together the probability mass of all values (from the respective curve) that are larger than the deadline. In this example task $\tau_{5}$ has a deadline of 28 . The probability that this deadline is missed is equal to $0.00935(\leq 0.01)$ in criticality mode $L_{1}$ of the system, to $0.00177(\leq 0.01)$ in mode $L_{2}$ and $0.00011(\leq 0.1)$ in mode $L_{3}$. The reason that $\tau_{5}$ has a smaller DMP in mode $L_{3}$ than in modes $L_{2}$ and $L_{1}$, even though it is allowed to have a larger DMP, is once more because the pWCET distributions are decreasing, making it highly unlikely for large response times to even be present in the system. If, on the contrary, we 


\begin{tabular}{|c|c|c|c|c|c|c|c|c|c|}
\hline ID & \multicolumn{6}{|c|}{ pWCET } & $\mathrm{T}$ & $\mathrm{D}$ & $\chi$ \\
\hline$\tau_{1}$ & $\begin{array}{c}1 \\
0.8\end{array}$ & $\begin{array}{c}2 \\
0.1\end{array}$ & $\begin{array}{c}3 \\
0.099\end{array}$ & $\begin{array}{c}4 \\
0.0009\end{array}$ & $\begin{array}{c}5 \\
0.00009\end{array}$ & $\begin{array}{c}6 \\
0.00001\end{array}$ & 10 & 10 & $L_{3}$ \\
\hline$\tau_{2}$ & $\begin{array}{c}1 \\
0.9\end{array}$ & $\begin{array}{l}3 \\
.09\end{array}$ & $\begin{array}{c}4 \\
.0099\end{array}$ & $\begin{array}{c}7 \\
0.00009\end{array}$ & $\begin{array}{c}8 \\
0.000009\end{array}$ & $\begin{array}{c}10 \\
0.000001\end{array}$ & 15 & 15 & $L_{2}$ \\
\hline$\tau_{3}$ & $\begin{array}{c}2 \\
0.7\end{array}$ & $\begin{array}{c}3 \\
0.199\end{array}$ & $\begin{array}{c}5 \\
0.01\end{array}$ & $\begin{array}{c}6 \\
0.05\end{array}$ & $\begin{array}{c}8 \\
0.04099\end{array}$ & $\begin{array}{c}9 \\
0.00001\end{array}$ & 20 & 20 & $L_{1}$ \\
\hline$\tau_{4}$ & $\begin{array}{c}3 \\
0.9\end{array}$ & $\begin{array}{c}4 \\
0.09\end{array}$ & $\begin{array}{c}5 \\
0.005\end{array}$ & $\begin{array}{c}7 \\
0.00399\end{array}$ & $\begin{array}{c}8 \\
0.001\end{array}$ & $\begin{array}{c}11 \\
0.00001\end{array}$ & 20 & 20 & $L_{3}$ \\
\hline$\tau_{5}$ & $\begin{array}{c}4 \\
0.9\end{array}$ & $\begin{array}{c}6 \\
0.09\end{array}$ & $\begin{array}{c}7 \\
0.009\end{array}$ & $\begin{array}{c}9 \\
0.0009\end{array}$ & $\begin{array}{c}10 \\
0.00009\end{array}$ & $\begin{array}{c}12 \\
0.00001\end{array}$ & 28 & 28 & $L_{2}$ \\
\hline
\end{tabular}

EXAMPLE OF A TASK-SET WITH PROBABILISTIC WORST CASE EXECUTION TIMES DISTRIBUTIONS.

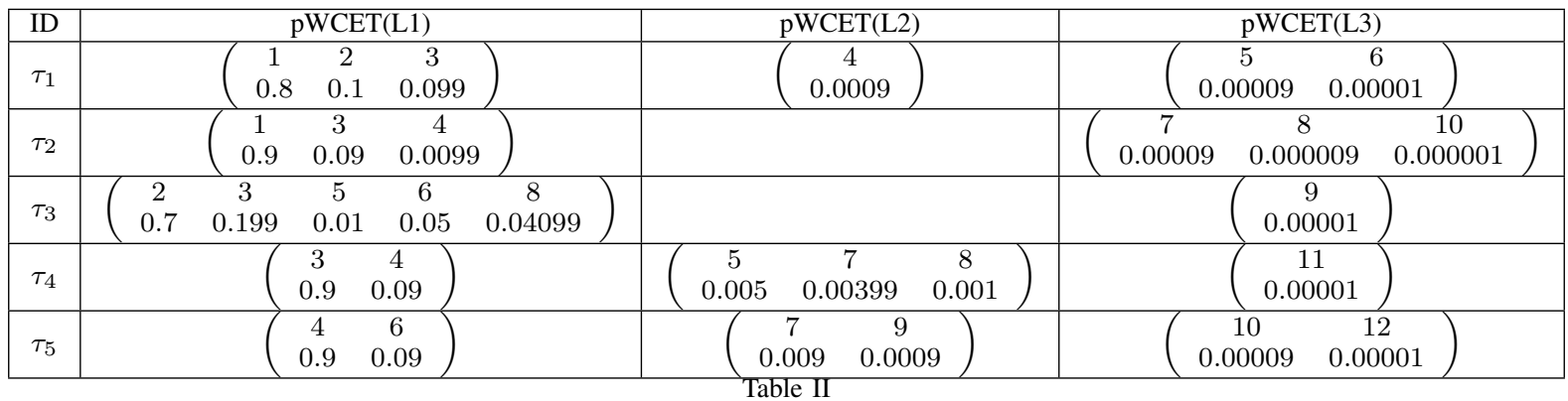

THE SPLITTING OF PWCETS INTO PARTIAL DISTRIBUTIONS ACCORDING TO THE CRITICALITY THRESHOLDS.

would have chosen increasing pWCETs for our example, then the curve representing mode $L_{3}$ would have been above the other two curves and also the tasks' DMP in mode $L_{3}$ would be much larger than in the other modes.

If we coalesce these three partial distributions we would obtain the complete response time distribution of the task independent of the functioning mode of the system. This would be the same distribution that the analysis of [6] would return. According to this analysis, the DMP of the task (irrespective of the systems criticality) would be 0.01124 which is larger than the threshold of 0.01 that is imposed on the task, hence the task would be deemed unschedulable at this priority level.

\section{CONCLUSION}

In this paper we proposed mixed criticality real-time system model and a probabilistic schedulability analysis for MCRTSs running on a single processor according to a fixed priority preemptive scheduling policy. The analysis extends the existing state of the art probabilistic analysis to the case of mixed criticalities, taking into account both the level of assurance at which each task needs to be certified, as well as the possible criticalities at which the system may execute.The proposed analysis is formally presented as well as explained with the aid of an illustrative example. As future work we plan to provide a formal proof of correctness. Intuitively the analysis is safe as it is a direct extension of an existing state of the art analysis which we further refine to decompose its results according to various functioning modes of the system.

\section{ACKNOWLEDGEMENTS}

This work was partially supported by the EU funded FP7 Integrated Project PROXIMA (611085), the FR BGLE funded Departs project (O16526-405635), the FR LEOC Capacites project, and the FR FUI Waruna project. The authors would like to thank Dagstuhl Seminar 15121 from which this work has emerged.

\section{REFERENCES}

[1] A. Burn, "Mixed criticality - a personal view," S. K. Baruah, L. CucuGrosjean, R. I. Davis, and C. Maiza, Eds., vol. 5, no. 3. Schloss Dagstuhl-Leibniz-Zentrum fuer Informatik, 2015.

[2] S. Vestal, "Preemptive scheduling of multi-criticality systems with varying degrees of execution time assurance," in the 28th IEEE RealTime Systems Symposium RTSS 2007, 2007.

[3] A. Burns and R. I. Davis, "Mixed criticality systems - a review [online]."

[4] T. Tia, Z. Deng, M. Shankar, M. Storch, J. Sun, L. Wu, and J. Liu, "Probabilistic performance guarantee for real-time tasks with varying computation times," in IEEE Real-Time and Embedded Technology and Applications Symposium ETFA 1995, 1995.

[5] M. Gardner and J. Lui, "Analyzing stochastic fixed-priority real-time systems," in the 5th International Conference on Tools and Algorithms for the Construction and Analysis of Systems TACAS 1999, 1999.

[6] J. Diaz, D. Garcia, K. Kim, C.-G. Lee, L. Lo Bello, J. Lopez, S. L. Min, and O. Mirabella, "Stochastic analysis of periodic real-time systems," in the 23rd IEEE Real-Time Systems Symposium RTSS 2002, 2002.

[7] Z. Guo, L. Santinelli, and K. Yang, "EDF schedulability analysis on mixed-criticality systems with permitted failure probability," in the $21 s t$ IEEE International Conference on Embedded and Real-Time Computing Systems and Applications RTCSA 2015, 2015.

[8] L. Santinelli and L. George, "Probabilities and mixed-criticalities: the probabilistic c-space," in the 3rd Workshop on Mixed Criticality Systems WMC 2015, 2015.

[9] L. Cucu-Grosjean, "Independence - a misunderstood property of and for (probabilistic) real-time systems," in "Real-Time Systems: the past, the present, and the future" conference organized in celebration of Professor Alan Burns sixtieth birthday, March 14th, 2013.

[10] L. Cucu-Grosjean, L. Santinelli, M. Houston, C. Lo, T. Vardanega, L. Kosmidis, J. Abella, E. Mezzetti, E. QuiÃśones, and F. J. Cazorla, "Measurement-based probabilistic timing analysis for multi-path programs," in the 24th Euromicro Conference on Real-Time Systems.

[11] D. Maxim and al., "Re-sampling for statistical timing analysis of realtime systems," in Proceedings of the 20th International Conference on Real-Time and Network Systems, 2012.

[12] A. G. Gogonel and L. Cucu-Grosjean, "How do we prove that probabilistic worst case response time is a Gumbel?" the 6th Real-Time Scheduling Open Problems Seminar, 2015. 